\title{
BMI percentile-for-age overestimates adiposity in early compared with late maturing pubertal children
}

\section{Kaspar Sørensen and Anders Juul}

Department of Growth and Reproduction, GR-5064, Copenhagen University Hospital, Blegdamsvej 9, DK-2100 Copenhagen, Denmark
Correspondence should be addressed to K Sørensen Email kasparsoerensen@gmail.com

\begin{abstract}
Objective: Early pubertal timing is consistently associated with increased BMI percentile-for-age in pubertal girls, while data in boys are more ambiguous. However, higher BMI percentile-for-age may be a result of the earlier puberty per se rather than vice versa. The aim was to evaluate markers of adiposity in relation to pubertal timing and reproductive hormone levels in healthy pubertal boys and girls.

Study design: Population-based cross-sectional study (The Copenhagen Puberty Study). Eight-hundred and two healthy Caucasian children and adolescents (486 girls) aged 8.5-16.5 years participated. BMI and bioelectric impedance analyses (BIA) were used to estimate adiposity. Clinical pubertal markers (Tanner stages and testicular volume) were evaluated. LH, FSH, estradiol, testosterone, SHBG and IGF1 levels were determined by immunoassays.

Results: In all age groups, higher BMI (all 1 year age-groups, $P \leq 0.041$ ) was found with early compared with late maturation, despite similar BIA-estimated body fat percentage (BIA-BF\%). Neither BMI nor BIA-BF\% differed for a given stage of maturation. BMI percentile-for-age and prevalence of overweight/obesity were higher in the early compared with late matured pubertal children (all $P \leq 0.038$ ), despite similar BIA-BF\%. Pubertal girls with BIA-BF $>29 \%$ had significantly lower LH and FSH levels compared with normal-weight girls $(P \leq 0.041)$.

Conclusions: Early maturational timing was not associated with higher adiposity for a given stage of puberty. Using BMI percentile-for-age overestimated the degree of adiposity in early pubertal compared with late pubertal children.
\end{abstract}

\section{Introduction}

Timing of sexual maturation is considered a marker of general public health (1). During recent decades, age at pubertal onset seems to have markedly declined in girls $(2,3,4,5,6)$ and to a lesser extent in boys $(6,7,8,9)$.

The increase in childhood adiposity over the last decades has received special attention as a possible etiological factor for the secular trends towards earlier onset of puberty $(10,11,12,13)$. That extremely low adiposity as seen with anorexia nervosa and extreme physical activity is associated with late pubertal timing is well established $(14,15)$. In contrast, to what extent
(C) 2015 European Society of Endocrinology Printed in Great Britain overweight and obesity is associated with early pubertal timing is more controversial.

Earlier age at breast development has consistently been associated with higher BMI percentile-for-age (Z-score) in girls $(11,12,16,17)$. Accordingly, a BMI $Z$-score in the overweight and obesity range is more prevalent in early compared with average to late maturing pubertal girls (11). In addition, higher BMI and skin fold thickness in infancy and childhood seem to predict earlier onset of breast development and menarche $(18,19,20,21)$. However, reproductive hormone levels have been reported

Published by Bioscientifica Ltd. 
to be lower in obese compared with normal-weight pubertal girls (22). Thus, inconsistency exists between the findings reported from the population-based studies relying solely on pubertal staging and studies reporting possible effects of obesity on the activation of the hypothalamic-pituitary-gonadal axis.

In boys, the prevalence of overweight and obesity has been reported to be higher in both late (11) and early maturing boys (17). In accordance with the latter, we recently found that the increase in BMI $Z$-scores over the past decades was associated with the coincident decline in age at puberty (8). However, the cause or effect relationship could not be determined in our study. In contrast to our previous findings, a follow-up on a subset of the US national population-based cohort, NHANES III, showed that being pre-pubertal at the age of 11.5 years was more prevalent in obese boys compared with boys with an average BMI $Z$-score in childhood (23). This finding is in accordance with studies reporting impaired gonadal function in obese pubertal boys $(24,25)$.

Most of the available population-based data on pubertal timing have used BMI Z-scores as a surrogate marker of adiposity. However, BMI increases with maturation even without changes in adiposity (26). Thus, BMI $Z$-scores, based on average BMI-for-age, will inevitably lead to overestimation of adiposity in pubertal children with early maturation and vice versa with late maturation. This important consideration has not been adequately addressed in previous studies reporting intra-pubertal differences in adiposity between early and late maturing children $(8,11,12,17)$.

The aim of the present study was to evaluate differences in adiposity between early and late maturing pubertal children in a cross-sectional population-based cohort of boys and girls. We evaluated BMI and adiposity by bioelectric impedance analysis (BIA) estimated body fat percentage (BF\%) and pubertal timing by clinical and hormonal markers.

\section{Subjects and Methods}

\section{Study subjects}

A total of 1894 healthy boys and girls (767 boys) aged 5.8-19.8 years participated in the Copenhagen Puberty Study that was conducted at schools in the Copenhagen area in 2006-2008. Other aspects of this study have previously been published $(4,8)$. In brief, all boys and girls from selected primary and high schools were invited to participate. The overall participation rates were 35.4 and $24.7 \%$ in girls and boys respectively. One hundred and sixty-five children ( 63 boys) with a condition potentially affecting pubertal development or of non-Caucasian descent were excluded based on questionnaire-collected information on previous medical history and parental ethnicity. To divide the cohort into early, average and late maturers, all girls from the original cohort aged 8.5-15.5 years $(n=670)$ and all boys aged $10.5-16.5$ years $(n=322)$ were selected.

The stratification into early, average and late maturers was based on the age at attainment of a certain pubertal milestone - defined by breast stages and menarche in girls and testicular volume (TV) in boys. It was assumed that the higher the breast stage or TV, the further the subject was in pubertal development.

A girl was defined as an early maturer if she reached a certain breast stage or menarche earlier than the median age for that stage in the population. A girl that reached a certain breast stage later than the median age for the next stage in development was defined as a late maturer and those in between - reaching a certain stage later than the medium of the stage, but before the medium age of the next stage was classified as an average maturer (4). For example, a girl would be classified as an early maturer if she was at breast stage 2 and her chronological age was less than the median age for breast stage 2 , or classified as a late maturer if she was in breast stage 2 and her chronological age was more than the median age for breast stage 3 . In addition, girls more than 13.0 years of age at examination were stratified by reported age at menarche (early $<13$ years; average $13-<13.5$ years and late $>13.5$ years). By this process, the girls we divided into early $(n=179)$, average $(n=149)$ and late maturers $(n=158)$. Pre-pubertal girls aged below the population mean for breast stage 2 $(n=118)$, girls lacking either breast examination $(n=7)$ or reported age at menarche after 13 years of age $(n=59)$ could not be classified and were excluded.

In boys, TV was used as the principal clinical pubertal marker in boys in accordance with expert panel recommendation (27). The TV 33rd and 67th percentile for age was used as a cut-off between the early $(n=87)$, average $(n=98)$ and late maturers $(n=131)$. Six boys were excluded due to lack of TV examination.

\section{Pubertal examination}

Pubertal stages (breast, genital and pubic hair) were evaluated by clinical examination including breast palpation according to the classification by Marshall \& Tanner $(28,29)$. TV was estimated in all boys by palpation to the 
nearest $1 \mathrm{ml}$ by orchidometry (30). In case TVs of the two testes were not equal, the larger testis measurement was used. Pubertal onset was defined as a TV of more than $3 \mathrm{ml}$ $(30,31)$. The same pediatric endocrinologist did all evaluations in girls and boys respectively. The pubertal period was defined as breast stages 2-4 or menarche in girls and TV of 4-19 $\mathrm{ml}$ in boys.

\section{Anthropometrics and body composition}

Standing height was measured to the nearest $0.1 \mathrm{~cm}$ using a portable stadiometer (Holtain Ltd, Crymych, UK). Weight was measured on a digital electronic scale (SECA delta, model 707, Hamburg, Germany) with a precision of $0.1 \mathrm{~kg}$. The children were weighed without shoes, wearing light clothing. BMI was calculated as weight (in kilograms) divided by height (in meters) squared. BMI $Z$-scores (SDS) were calculated according to the CDC2000 reference (32). Overweight was defined as BMI percentile-for-age $>85$ according to CDC2000 reference. Estimation of fat-free mass (FFM) was done by BIA (Holtain Ltd) using equations based on dual energy X-ray absorptiometry (DXA) scans (boys: $\mathrm{FFM}=-0.79+$ height $^{2} / Z \times 0.44+$ weight $\times 0.36$ $(r=0.99)$ and girls: $\mathrm{FFM}=0.27+$ height $^{2} / Z \times 0.39+$ weight $\times 0.38(r=0.99))$ previously described on a subgroup of the children (99 girls and 65 boys) (33). From estimations of FFM, fat mass (FM) (weight-FFM) and BIA-BF\% (FM/weight) were calculated.

\section{Laboratory analysis}

Blood samples were withdrawn from an antecubital vein between 0830 and $1300 \mathrm{~h}$, and were available in 264 boys and 408 girls. Blood samples were clotted, centrifuged, and serum was stored at $-20{ }^{\circ} \mathrm{C}$ until hormone analyses were performed. Serum follicle-stimulating hormone (FSH), luteinizing hormone $(\mathrm{LH})$, testosterone, estradiol $\left(\mathrm{E}_{2}\right)$, sex hormone-binding globulin (SHBG) and insulin-like growth factor 1 (IGF1) levels were measured by commercially available immune-assays as previously published $(4,8,34,35)$.

\section{Statistical analyses}

Data were cross-sectional. Statistical differences in body composition and reproductive hormone levels between the groups of early, average and late matured girls were evaluated in the 1-year age intervals and within each breast stage. In boys, similar analyses were done in 1-year age intervals as well as in TV-intervals (4-8; 9-13 and
14-19 ml). Differences between early and late maturers were done by Student's $t$-tests within each age and pubertal group. Adjusted analyses were done by general linear models (ANOVA). Differences in prevalence of overweight and obesity were evaluated by Fisher's exact tests. Associations between BIA-BF\%, BMI and BMI Z-scores for girls in breast stage 2 were done by linear regression analyses. The mean BIA-BF\% at classification of overweight and obesity was evaluated by probit analyses. All results are presented by means and 95\% CIs if not otherwise stated.

\section{Ethical considerations}

The Copenhagen Puberty Study (ClinicalTrials.gov \#NCT01411527) was approved by the Local Ethical Committee (\#KF 01282214 and \#V200.1996/90) and conducted in accordance with the Second Helsinki Declaration. All participants and their parents gave informed consent.

\section{Results}

\section{Girls}

In all girls, age was positively associated with weight, height, BMI, BIA-FFM and BIA-FM (all $P \leq 0.015$ ), but not with BIA-BF\% $(P=0.28)$ respectively. Similar associations were found with breast stages (all $P \leq 0.001$ ). The differences in anthropometrics, adiposity and reproductive hormone levels between the early matured, the average and the late matured girls stratified into 1-year age intervals are shown in Fig. 1. When stratified according to maturation, the early matured girls were significantly younger with lower stature and weight (all $P<0.003)$, but with no differences in BMI, BIA-FM or $\mathrm{BIA}-\mathrm{BF} \%$ at a given breast stage compared with the late matured girls. BMI $Z$-scores was significantly higher in the group of early matured compared with the late matured girls for a given breast stage ( $\Delta 0.91$ s.D. $(0.65-$ 1.17), $P<0.001$, ANOVA; Fig. 2). Additional adjustment for BIA-BF\% did only marginally change this finding ( $\Delta 0.72$ s.D. (0.53-0.91), $P<000.1)$. The prevalence of overweight and obesity were significantly higher in the group of early matured compared with the late matured girls during puberty $(16.4 \%$ vs $6.3 \%, P=0.025)$. The mean BIA-BF\% at classification of overweight was $29.0 \%$ (28.3-30.0) in girls. The early matured girls were classified as being overweight at a borderline significantly lower BIA-BF\% compared with late matured girls during the pubertal period ( $\triangle \mathrm{BIA}-\mathrm{BF} \% 1.99 \%(-0.07$ to 4.62$)$, 

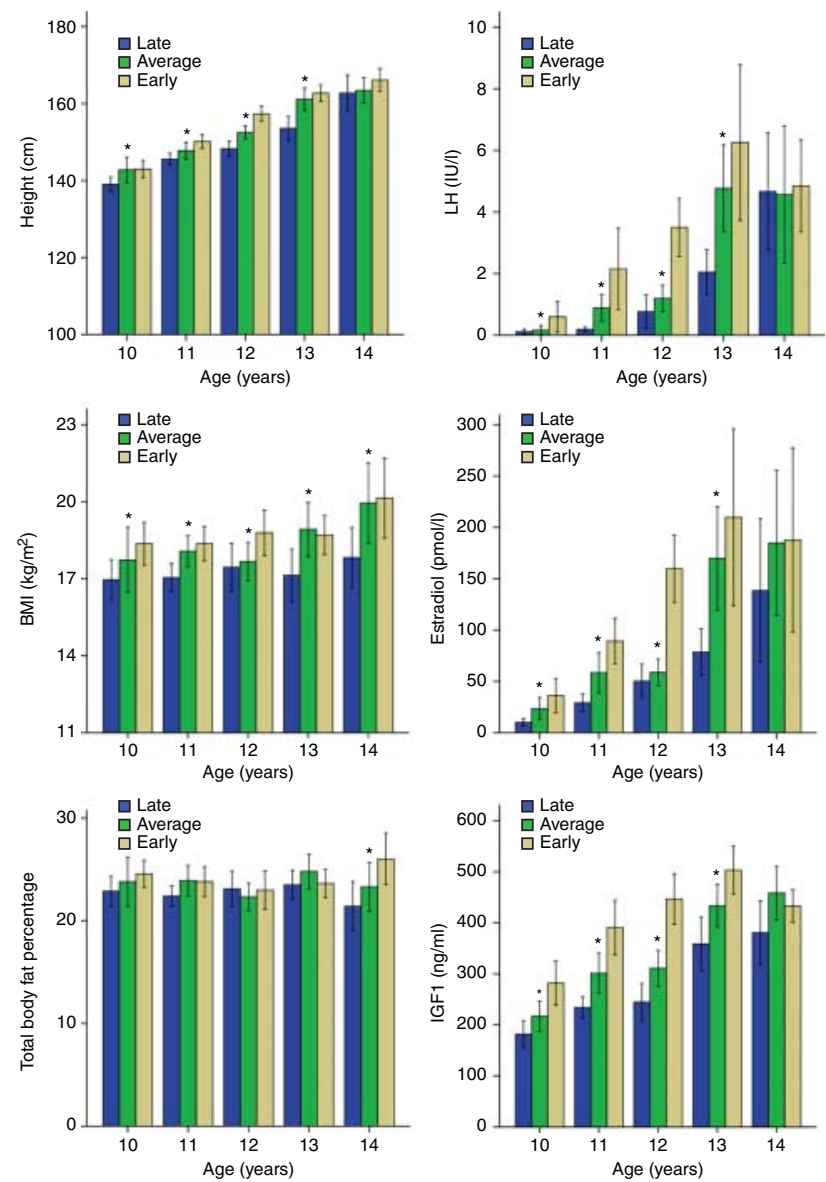

\section{Figure 1}

Height, BMI, total body fat percentage by bioelectric impedance analysis (BIA-BF\%) as well as luteinizing hormone ( $\mathrm{LH})$, estradiol and insulin-like growth factor 1 (IGF1) levels in 486 girls stratified by 1 -year age intervals and divided into late (blue bars), average (green bars) and late matured (grey bars). Data are presented as means and $95 \% \mathrm{Cls}$ (error bars). Differences between early and late matured girls by Student's $t$-tests $(* P<0.05)$.

$P=0.062$ ). The prevalence of having a BIA-BF\% $>29 \%$ was not different between early and late matured girls (8.6\% vs $5.1 \%, P=0.25)$.

Age at breast stage 2 was significantly inversely associated with BMI $\left(-0.29 \mathrm{~kg} / \mathrm{m}^{2}\right.$ per year $(-0.51$ to -0.08$), P=0.009)$, BMI $Z$-score $(-0.42 \mathrm{SDS} /$ year $(-0.57$ to -0.26$), P<0.001)$ and BIA-BF\% $(-0.9 \% / y e a r$ $(-1.7$ to -0.1$), P=0.024)$. Despite additional adjustment for $\mathrm{BIA}-\mathrm{BF} \%$, the BMI $Z$-score remained strongly inversely associated with age at breast stage $2(-0.27 \mathrm{SDS} /$ year $(-0.39$ to -0.15$), P<0.001)$, while association to BMI disappeared $\left(-0.02 \mathrm{~kg} / \mathrm{m}^{2}\right.$ per year $(-0.32$ to 0.27$\left.), P=0.88\right)$.
In breast stages 2 and 3, LH and $\mathrm{E}_{2}$ levels were higher in the late pubertal compared with the early pubertal group (all $P \leq 0.026$ ), with no significant differences in SHBG ( $P \geq 0.10)$. IGF1 levels were higher in late compared with early matured girls only in breast stage $3(P=0.027)$. Lower SHBG, FSH and LH levels (all $P \leq 0.041$ ) was found in girls with $\mathrm{BIA}-\mathrm{BF} \%>29 \%$ compared with the rest of the pre-menarchal pubertal girls. Classification as overweight/obese by CDC2000 was associated with lower
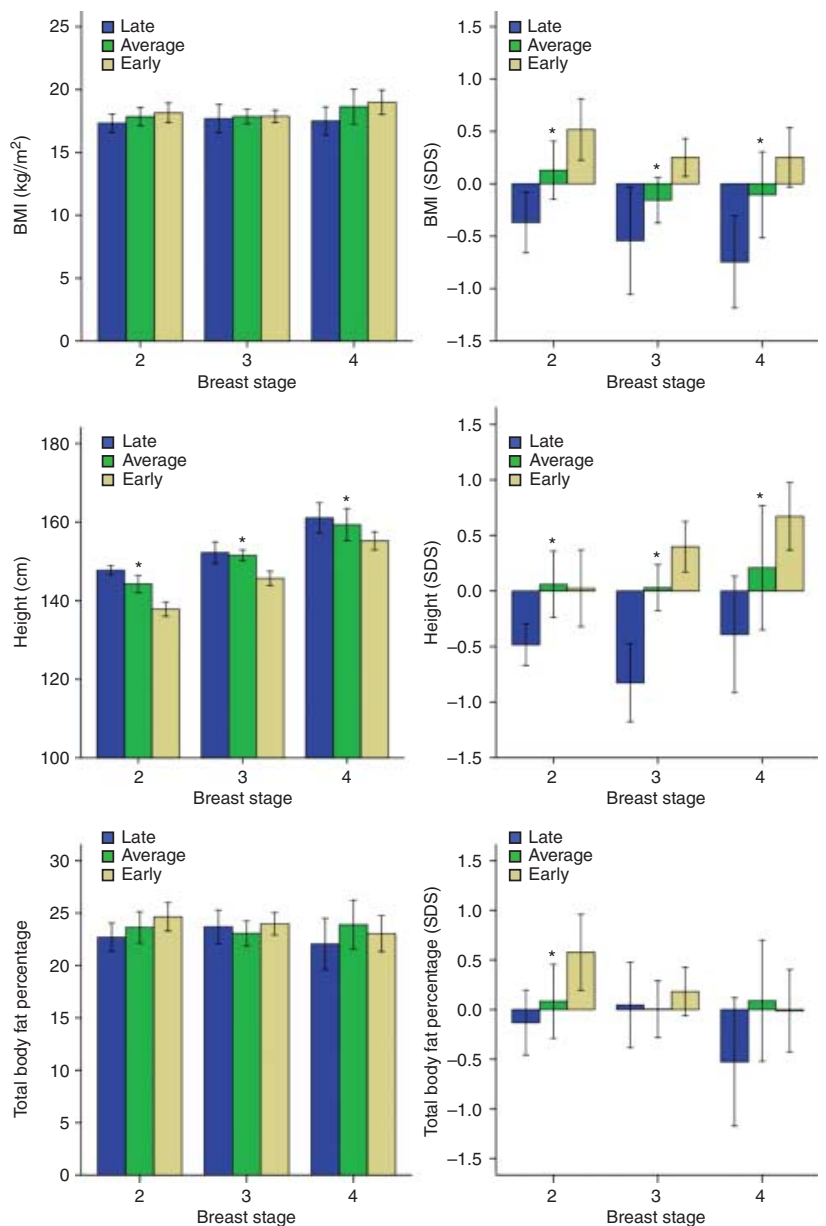

\section{Figure 2}

BMI, BMI Z-score (SDS for age according to the CDC2000 reference), height, height-SDS, total body fat percentage (by bioelectrical impedance analysis (BIA-BF\%)) and BIA-BF\%-SDS in relation to breast stages 2-4 in 335 pre-menarcheal pubertal girls divided into groups of early (blue bars), average (green bars) and late maturation (grey bars). Data are presented as means and $95 \% \mathrm{Cls}$ (error bars). Differences between early and late matured girls by Student's $t$-tests $\left({ }^{*} P<0.05\right)$. Mean age was higher in late compared with early matured girls within each breast stage (all $P<0.001$ ). 
SHBG levels $(P<0.001)$ but with no other differences in reproductive hormone levels ( $\mathrm{E}_{2}, \mathrm{FSH}, \mathrm{LH}$ and IGF1).

\section{Boys}

Weight, height, BMI, BIA-FFM and BIA-FM was positively, and BIA-BF\% negatively, associated with age in all boys (all $P \leq 0.015$ ). Similar associations were found with TV (all $P \leq 0.001)$, except for BIA-FM $(P=0.26)$. The differences in anthropometrics, adiposity and reproductive hormone levels between the early, the average and the late matured boys stratified into 1-year age intervals are shown in Fig. 3.
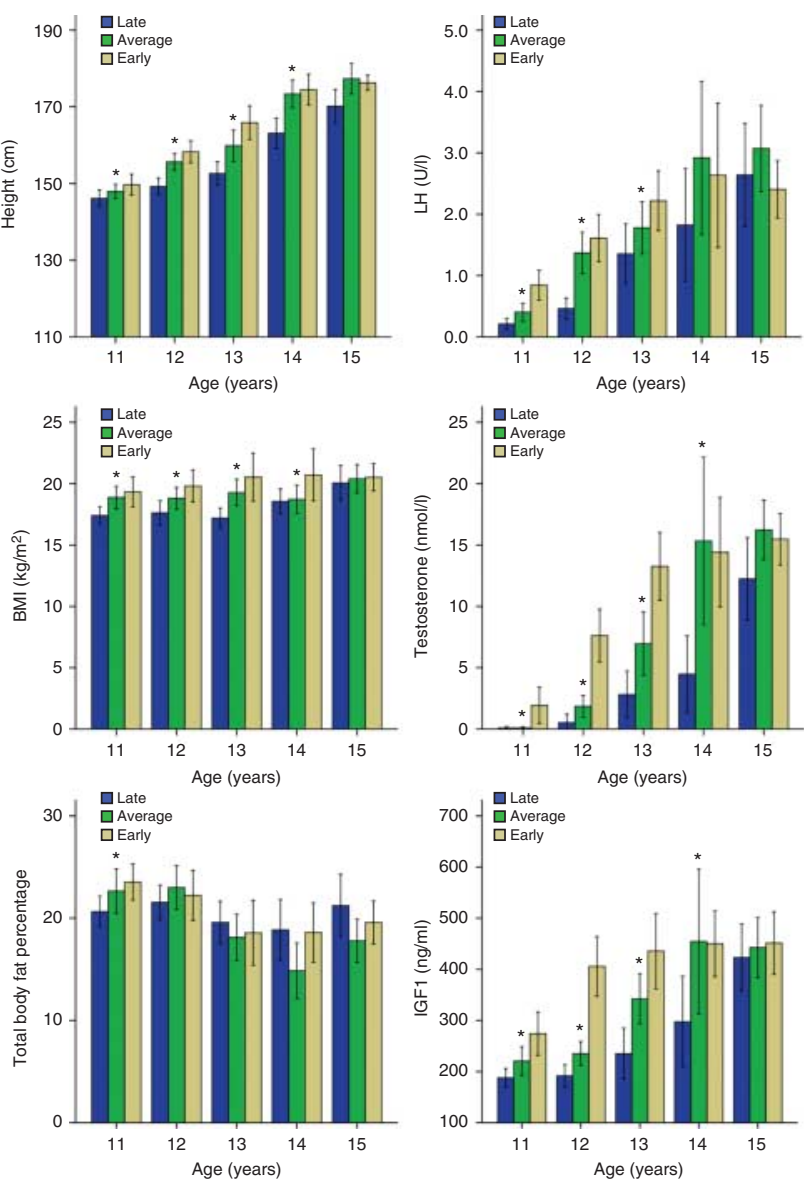

Figure 3

Height, BMI, total body fat percentage by bioelectric impedance analysis (BIA-BF\%) as well as testosterone, luteinizing hormone (LH) and insulin-like growth factor 1 (IGF1) levels in 316 boys stratified by 1-year age intervals and divided into late (blue bars), average (green bars) and late matured (grey bars). Data are presented as means and $95 \%$ Cls (error bars).

Differences between early and late matured boys by Student's t-tests $\left({ }^{*} P<0.05\right)$.
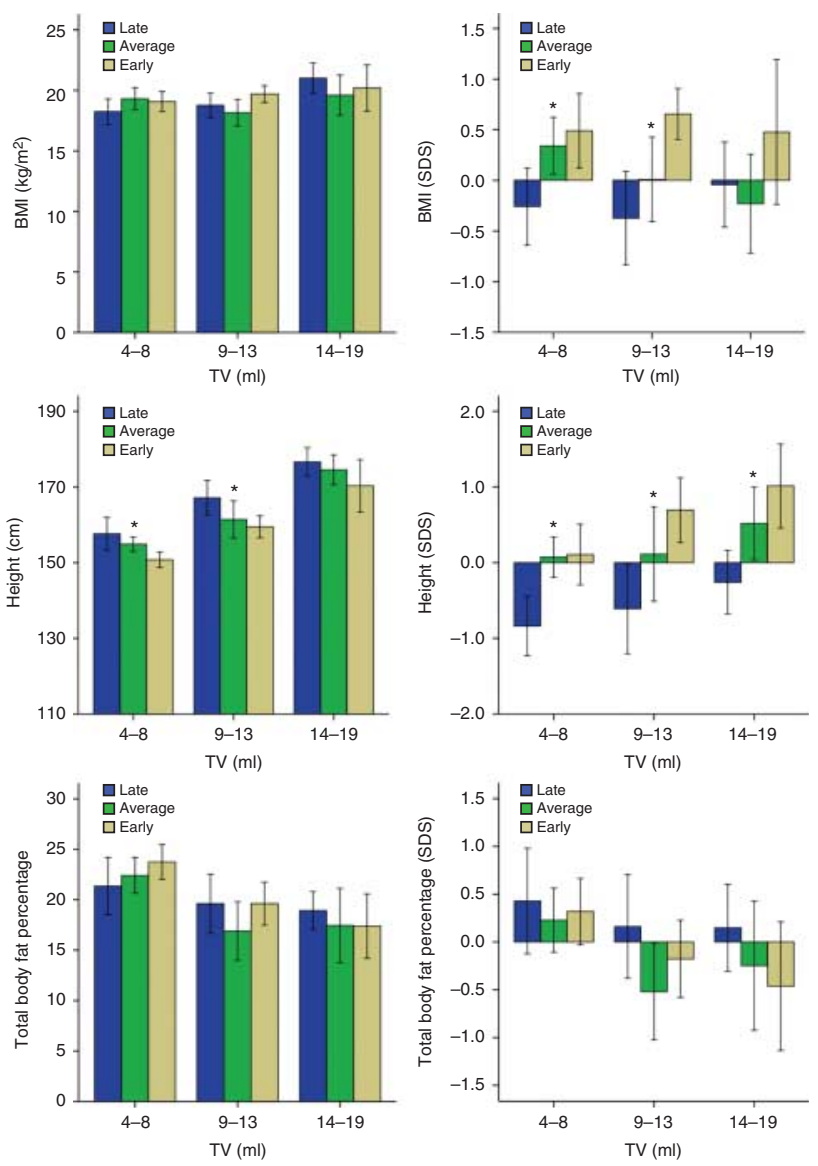

Figure 4

BMI, BMI Z-score (SDS for age according to the CDC2000 reference), height, height-SDS, total body fat percentage (by bioelectrical impedance analysis (BIA-BF\%)) and BIA-BF\%-SDS in relation to testicular volume (TV) groups in 175 pubertal boys divided into groups of early (blue bars), average (green bars) and late maturation (grey bars). Data are presented as means and $95 \% \mathrm{Cls}$ (error bars). Differences between early and late matured boys by Student's $t$-tests ( $\left.{ }^{*} P<0.05\right)$. Mean age was higher in late compared with early matured boys within each TV group (all $P<0.001$ ).

The early matured boys were significantly younger, shorter and with lower BIA-FFM compared with both average and late matured boys at a given TV (all $P \leq 0.042$ ), but with no significant differences in weight, BMI, BIA-FM and BIA-BF\% (all $P \geq 0.086$ ). However, the mean BMI $Z$-score was significantly higher in the group of early matured compared with the late matured boys for a given TV ( $\Delta 0.75$ s.D. (0.43-1.06), $P<000.1$; Fig. 4). Additional adjustment for BIA-BF\% did only marginally change this finding ( $\Delta 0.58$ s.D. (0.32-0.85), $P<000.1)$. The prevalence 
of overweight and obesity (BMI Z-score $>85$ th percentile) during the pubertal period was higher in the early compared with the late matured boys $(24.5 \%$ vs $10.6 \%$, $P=0.038$ ). The mean BIA-BF\% at overweight was $28.1 \%$ (26.4-31.1) in all boys. The early matured boys were classified as being overweight at a significantly lower BIABF\% compared with late matured boys ( $\triangle \mathrm{BIA}-\mathrm{BF} \% 2.98 \%$ (0.01-7.09), $P=0.018)$. The prevalence of having a BIA-BF $>28 \%$ was similar between early and late matured pubertal boys $(7.5 \%$ vs $9.1 \%, P=0.52)$.

No differences in reproductive hormone levels (testosterone, LH, FSH, SHBG and IGF1) were found between the early, the average, and the late matured boys in the pubertal period when stratified for TV (all $P \geq 0.15$ ). Reproductive hormone levels were not significantly different in those with BIA-BF\% $>28 \%$ or overweight/ obesity classified by CDC2000 compared with the rest of the cohort within each TV group (all $P>0.08$ ), except for lower SHBG levels in the 4-9 $\mathrm{ml} \mathrm{TV}$ group (both $P<0.001$ ).

\section{Discussion}

In the present population-based study of healthy children and adolescents, early matured boys and girls had higher BMI, but similar BF\%, compared with late matured similarly aged girls and boys respectively. Despite no differences in either BMI or adiposity, higher BMI Z-scores for a given stage of puberty were found in the early matured compared with the late matured boys and girls respectively. The prevalence of overweight and obesity was significantly higher in early compared with late matured pubertal children, despite similar prevalence of high BIA-BF\%. Thus, BMI percentile-for-age (Z-scores) overestimate adiposity in early compared with late maturing children.

The recent secular increase in adiposity has received special attention as a potential etiological factor related to the secular changes towards earlier age at pubertal onset. In accordance with previous population-based studies $(8,11,12)$, we found that early maturing children have higher BMI compared with similarly aged late maturing children. However, BMI increases with the maturational increase in stature and total body mass even with stable or decreasing adiposity during puberty as shown in both the present and previous studies (26). Thus, early maturing pubertal children are expected to have higher BMI for a given age compared late maturing children due to earlier age at pubertal growth spurt and accretion of both lean and FM. Thus, higher BMI do not reflect higher adiposity per se. In accordance, no significant differences were found in total BF\% between similarly aged early and late maturing children.

To account for the marked changes in body composition during puberty, it is more appropriate to evaluate differences in body composition between early and late matured children at the same stage of maturation. When stratified for breast stage in girls and TV in boys, differences were found in neither BMI nor BF\% between the early and late maturing children. Thus, the higher BMI-for-age in the early maturing children seem to be explained by the earlier age at puberty per se. Early maturing pubertal children may be viewed as being closer to adult BMI at an earlier age than their less matured peers. Thus, simply adjusting BMI for age leads to overestimation of adiposity in early compared with late maturing children. In analogy, early maturing pubertal children is taller - being closer to final-height - than their similarly aged less matured peers due to earlier age at growth acceleration. In other words, they do not end up being tall statured as the height $Z$-score may imply during the pubertal period. This is a well-recognized clinical issue generally overcome by relating height for bone-age, thereby adjusting height for stage of maturation. These important considerations have not been taking into account in previously published studies on intra-pubertal differences in BMI - many of which have used age-adjusted BMI (Z-scores) a surrogate marker of adiposity (10, 11, $12,13)$. In the present study, the early maturing children had markedly higher BMI $Z$-scores for a given stage of puberty despite similar actual BMI and total BF\%. Thus, the inappropriate age-adjustment of BMI made these early pubertal girls seem more adipose. The lack of acknowledgement of this maturational effect on BMI may at least to some extent have overestimated BMI in early pubertal children reported in most previous published population-based cohort studies $(8,11,12,17)$. In the study by Kaplowitz et al. (12), the BMI Z-score was $0.25-0.50$ s.D. higher per year earlier age at breast stage 2 . In a similar analysis, we found that the BMI Z-score increased by 0.27 s.D./year earlier age at breast stage 2 , after adjustment for adiposity.

Although the maturational effect on BMI most likely diminished with increasing adiposity, it seems to influence classification of overweight when based on BMI percentile-for-age. In the present study, higher prevalence of overweight and obesity were found in early compared with late matured pubertal girls, in accordance with most previous studies $(11,12)$. However, the prevalence of high adiposity (total body fat $>29 \%$ ) was not significant different between the early and late matured girls. Thus, 
using BMI $Z$-scores also seem to overestimate the prevalence of overweight in early compared with late matured pubertal girls. Our findings are further supported by studies showing lower gonadotropin and $\mathrm{E}_{2}$ levels in early-to-mid-pubertal obese girls compared with similarly matured normal-weight girls (22). In accordance, we found that pubertal girls with adiposity $>29 \%$ had lower gonadotropin levels compared with the normal-weight pubertal girls. Thus, high adiposity does not seem to be associated with a more potent activation of the hypothalamic-pituitary-gonadal axis in girls, rather vice versa. The findings of more advanced breast development in overweight and obese girls in population-based studies $(11,12)$ may be partly explained by over-classification of breast stages in these girls due to the mimicking lipomastia, which should be kept in mind in the evaluation of breast stages in girls with excess adiposity. In addition, gonadotropin-independent effects of adiposity-related hormones on glandular breast development may play a role (36). In theory, lower SHBG levels may increase the bioavailability of sex steroid hormones to glandular breast tissue (37). In the present study, lower SHBG levels were found in the girls classified as overweight/obese by both BMI and BIA as previously reported (38).

Higher prevalence of overweight and obesity, based on BMI percentile-for-age, was also found in the early compared late matured pubertal boys, despite similar prevalence of total body fat above $28 \%$. This discrepancy seem to be related to fact that the early matured pubertal boys was classified as overweight at a significantly lower BF\% compared with the late maturing pubertal boys, when classification was based on BMI percentile-for-age (32). Thus, the findings of higher prevalence of overweight in early maturing boys in previous European studies (17) may partly be biased by the use of ageadjusted BMI for classification of overweight in pubertal boys. In contrast to the European studies, the American population-based study, NHANES III, reported that overweight and obesity was less prevalent in boys with early compared to average to late puberty (11). These findings was later supported in a longitudinal follow-up of the NHANES III (23). The cross-Atlantic discrepancies may be related to a higher degree of adiposity in the American population-based studies, thereby reducing the maturational effect on BMI as well as evaluating a more severe obesity phenotype. In accordance with less advanced signs of puberty with increasing adiposity, lower testosterone and gonadotropin levels have previously been reported in obese pubertal boys $(24,25)$. Although similar findings could not be demonstrated in the present study, it is plausible that obesity can impair gonadal function in pubertal boys as has been reported in obese adults $(39,40)$.

Our present results indicate that the differences in adiposity between early and late matured girls may not be as pronounced as previously reported. However, prepubertal adiposity may still influence pubertal timing. In favour of such a relationship, several studies have found that early maturing children have thicker skin folds and larger body size during infancy and early childhood as well as greater increases in skin fold thickness and body size before puberty compared with later matured children (18, $19,20,21)$. Despite the limitation of the cross-sectional design, the present study is the first to adequately describe the limitation of BMI percentile-for-age as a surrogate marker of normal and excess adiposity when evaluating children differing in pubertal timing.

BIA is a cost-effective, rapid and non-invasive method to estimate body composition in children and adolescents. Although inferior to laboratory-based techniques such as isotope dilution, hydrodensitometry, DXA and magnetic resonance imaging, the use of BIA for evaluation of body composition in children and adolescents is generally considered to be more accurate than anthropometric-based equations such as BMI (41). BIA estimation is based on the assumption that the body is a cylindrical ionic conductor. In addition to the intra- and extracellular fat-free compartments, conductance depends on the length and calibre of the body cylinders, which may change markedly during puberty. In accordance, the precision of BIA in estimating body composition seems to vary across the peri-pubertal period (42). Thus, even with the use of BIA it seems most appropriate to evaluate body composition for a given stage of puberty rather than age.

In conclusion, early pubertal timing was not associated with higher adiposity within the pubertal period in either girls or boys. Despite lack of significant differences in adiposity, the BMI percentile-for-age and the prevalence of overweight was significantly higher in early maturing compared with late maturing children for the same stage of maturation. Thus, BMI percentile-for-age seems to overestimate adiposity and classification of overweight in early compared with late maturing children during the pubertal period. Our results indicate that adjustment of BMI for stage of maturation rather than age will improve the accuracy in the evaluation of adiposity between early and late maturing children during the pubertal period. 


\section{Declaration of interest}

The authors declare that there is no conflict of interest that could be perceived as prejudicing the impartiality of the research reported.

\section{Funding}

The first draft of the manuscript was written by K Sørensen. No honorarium, grant or other form of payment was given to anyone to produce the manuscript.

\section{Acknowledgements}

We would like to thank the all the participants, parents, teachers and principals of the participating schools. The Copenhagen Puberty Study was kindly supported by the Kirsten and Freddy Johansen Foundation and by The Danish Medical Research council (\#271-05-0337).

\section{References}

1 Golub MS, Collman GW, Foster PM, Kimmel CA, Rajpert-De Meyts E, Reiter EO, Sharpe RM, Skakkebaek NE \& Toppari J. Public health implications of altered puberty timing. Pediatrics 2008121 (Suppl 3) S218-S230. (doi:10.1542/peds.2007-1813G)

2 Herman-Giddens ME, Slora EJ, Wasserman RC, Bourdony CJ, Bhapkar MV, Koch GG \& Hasemeier CM. Secondary sexual characteristics and menses in young girls seen in office practice: a study from the Pediatric Research in Office Settings network. Pediatrics 199799 505-512. (doi:10.1542/peds.99.4.505)

3 Sun SS, Schubert CM, Chumlea WC, Roche AF, Kulin HE, Lee PA, Himes JH \& Ryan AS. National estimates of the timing of sexual maturation and racial differences among US children. Pediatrics 2002 110 911-919. (doi:10.1542/peds.110.5.911)

4 Aksglaede L, Sorensen K, Petersen JH, Skakkebaek NE \& Juul A. Recent decline in age at breast development: the Copenhagen Puberty Study. Pediatrics 2009123 e932-e939. (doi:10.1542/peds.2008-2491)

5 Biro FM, Galvez MP, Greenspan LC, Succop PA, Vangeepuram N, Pinney SM, Teitelbaum S, Windham GC, Kushi LH \& Wolff MS. Pubertal assessment method and baseline characteristics in a mixed longitudinal study of girls. Pediatrics 2010126 e583-e590. (doi:10.1542/peds.2009-3079)

6 Sorensen K, Mouritsen A, Aksglaede L, Hagen CP, Mogensen SS \& Juul A. Recent secular trends in pubertal timing: implications for evaluation and diagnosis of precocious puberty. Hormone Research in Paediatrics 201277 137-145. (doi:10.1159/000336325)

7 Karpati AM, Rubin CH, Kieszak SM, Marcus M \& Troiano RP. Stature and pubertal stage assessment in American boys: the 1988-1994 Third National Health and Nutrition Examination Survey. Journal of Adolescent Health 200230 205-212. (doi:10.1016/S1054-139X(01)00320-2)

8 Sorensen K, Aksglaede L, Petersen JH \& Juul A. Recent changes in pubertal timing in healthy Danish boys: associations with body mass index. Journal of Clinical Endocrinology and Metabolism 201095 263-270. (doi:10.1210/jc.2009-1478)

9 Herman-Giddens ME, Steffes J, Harris D, Slora E, Hussey M, Dowshen SA, Wasserman R, Serwint JR, Smitherman L \& Reiter EO. Secondary sexual characteristics in boys: data from the Pediatric Research in Office Settings network. Pediatrics 2012130 e1058-e1068. (doi:10.1542/peds.2011-3291)

10 Kaplowitz PB. Link between body fat and the timing of puberty. Pediatrics 2008121 (Suppl 3) S208-S217. (doi:10.1542/peds.2007. 1813F)
11 Wang Y. Is obesity associated with early sexual maturation? A comparison of the association in American boys versus girls Pediatrics 2002110 903-910. (doi:10.1542/peds.110.5.903)

12 Kaplowitz PB, Slora EJ, Wasserman RC, Pedlow SE \& HermanGiddens ME. Earlier onset of puberty in girls: relation to increased body mass index and race. Pediatrics 2001108 347-353. (doi:10.1542/peds. 108.2.347)

13 Slyper AH. The pubertal timing controversy in the USA, and a review of possible causative factors for the advance in timing of onset of puberty. Clinical Endocrinology 200665 1-8. (doi:10.1111/j.1365-2265.2006. 02539.x)

14 Warren MP. The effects of exercise on pubertal progression and reproductive function in girls. Journal of Clinical Endocrinology and Metabolism 198051 1150-1157. (doi:10.1210/jcem-51-5-1150)

15 Munoz MT \& Argente J. Anorexia nervosa in female adolescents: endocrine and bone mineral density disturbances. European Journal of Endocrinology 2002147 275-286. (doi:10.1530/eje.0.1470275)

16 Foster TA, Voors AW, Webber LS, Frerichs RR \& Berenson GS. Anthropometric and maturation measurements of children, ages 5 to 14 years, in a biracial community - the Bogalusa Heart Study. American Journal of Clinical Nutrition 197730 582-591.

17 Ribeiro J, Santos P, Duarte J \& Mota J. Association between overweight and early sexual maturation in Portuguese boys and girls. Annals of Human Biology 200633 55-63. (doi:10.1080/00207390500434135)

18 Davison KK, Susman EJ \& Birch LL. Percent body fat at age 5 predicts earlier pubertal development among girls at age 9. Pediatrics $2003 \mathbf{1 1 1}$ 815-821. (doi:10.1542/peds.111.4.815)

19 Lee JM, Appugliese D, Kaciroti N, Corwyn RF, Bradley RH \& Lumeng JC. Weight status in young girls and the onset of puberty. Pediatrics 2007 119 e624-e630. (doi:10.1542/peds.2006-2188)

20 Boyne MS, Thame M, Osmond C, Fraser RA, Gabay L, Reid M \& Forrester TE. Growth, body composition, and the onset of puberty: longitudinal observations in Afro-Caribbean children. Journal of Clinical Endocrinology and Metabolism 201095 3194-3200. (doi:10.1210/jc. 2010-0080)

21 Ong KK, Emmett P, Northstone K, Golding J, Rogers I, Ness AR, Wells JC $\&$ Dunger DB. Infancy weight gain predicts childhood body fat and age at menarche in girls. Journal of Clinical Endocrinology and Metabolism 200994 1527-1532. (doi:10.1210/jc.2008-2489)

22 McCartney CR, Prendergast KA, Blank SK, Helm KD, Chhabra S \& Marshall JC. Maturation of luteinizing hormone (gonadotropinreleasing hormone) secretion across puberty: evidence for altered regulation in obese peripubertal girls. Journal of Clinical Endocrinology and Metabolism 200994 56-66. (doi:10.1210/jc.2008-1252)

23 Lee JM, Kaciroti N, Appugliese D, Corwyn RF, Bradley RH \& Lumeng JC. Body mass index and timing of pubertal initiation in boys. Archives of Pediatrics \& Adolescent Medicine 2010164 139-144. (doi:10.1001/ archpediatrics.2009.258)

24 Denzer C, Weibel A, Muche R, Karges B, Sorgo W \& Wabitsch M. Pubertal development in obese children and adolescents. International Journal of Obesity 200731 1509-1519. (doi:10.1038/sj.ijo.0803691)

25 Taneli F, Ersoy B, Ozhan B, Calkan M, Yilmaz O, Dinc G, Genc A \& Taneli C. The effect of obesity on testicular function by insulin-like factor 3, inhibin B, and leptin concentrations in obese adolescents according to pubertal stages. Clinical Biochemistry 201043 1236-1240. (doi:10.1016/j.clinbiochem.2010.07.026)

26 Veldhuis JD, Roemmich JN, Richmond EJ, Rogol AD, Lovejoy JC, Sheffield-Moore M, Mauras N \& Bowers CY. Endocrine control of body composition in infancy, childhood, and puberty. Endocrine Reviews 200526 114-146. (doi:10.1210/er.2003-0038)

27 Euling SY, Herman-Giddens ME, Lee PA, Selevan SG, Juul A, Sørensen TI, Dunkel L, Himes JH, Teilmann G \& Swan SH. Examination of US puberty-timing data from 1940 to 1994 for secular trends: panel findings. Pediatrics 2008121 (Suppl 3) S172-S191. (doi:10.1542/peds. 2007-1813D) 
28 Marshall WA \& Tanner JM. Variations in the pattern of pubertal changes in boys. Archives of Disease in Childhood 197045 13-23. (doi:10.1136/adc.45.239.13)

29 Marshall WA \& Tanner JM. Variations in pattern of pubertal changes in girls. Archives of Disease in Childhood 196944 291-303. (doi:10.1136/ adc.44.235.291)

30 Zachmann M, Prader A, Kind HP, Hafliger H \& Budliger H. Testicular volume during adolescence. Cross-sectional and longitudinal studies. Helvetica Paediatrica Acta 197429 61-72.

31 Biro FM, Lucky AW, Huster GA \& Morrison JA. Pubertal staging in boys. Journal of Pediatrics 1995127 100-102. (doi:10.1016/S00223476(95)70265-2)

32 Ogden CL, Kuczmarski RJ, Flegal KM, Mei Z, Guo S, Wei R, GrummerStrawn LM, Curtin LR, Roche AF \& Johnson CL. Centers for Disease Control and Prevention 2000 growth charts for the United States: improvements to the 1977 National Center for Health Statistics version. Pediatrics 2002109 45-60. (doi:10.1542/peds.109.1.45)

33 Munch-Andersen T, Sorensen K, Andersen LB, Aachmann-Andersen NJ, Aksglaede L, Juul A \& Helge JW. Adverse metabolic risk profiles in Greenlandic Inuit children compared to Danish children. Obesity 2013 21 1226-1231. (doi:10.1002/oby.20203)

34 Sorensen K, Aksglaede L, Petersen JH, Andersson AM \& Juul A. Serum IGF1 and insulin levels in girls with normal and precocious puberty. European Journal of Endocrinology 2012166 903-910. (doi:10.1530/ EJE-12-0106)

35 Sorensen K, Aksglaede L, Petersen JH, Leffers H \& Juul A. The exon 3 deleted growth hormone receptor gene is associated with small birth size and early pubertal onset in healthy boys. Journal of Clinical Endocrinology and Metabolism 201095 2819-2826. (doi:10.1210/jc. 2009-2484)
36 Berryhill GE, Gloviczki JM, Trott JF, Aimo L, Kraft J, Cardiff RD, Paul CT, Petrie WK, Lock AL \& Hovey RC. Diet-induced metabolic change induces estrogen-independent allometric mammary growth. PNAS 2012109 16294-16299. (doi:10.1073/pnas.1210527109)

37 Mendel CM. The free hormone hypothesis: a physiologically based mathematical model. Endocrine Reviews 198910 232-274. (doi:10.1210/edrv-10-3-232)

38 Sorensen K, Andersson AM, Skakkebaek NE \& Juul A. Serum sex hormone-binding globulin levels in healthy children and girls with precocious puberty before and during gonadotropin-releasing hormone agonist treatment. Journal of Clinical Endocrinology and Metabolism 200792 3189-3196. (doi:10.1210/jc.2007-0231)

39 Pitteloud N, Hardin M, Dwyer AA, Valassi E, Yialamas M, Elahi D \& Hayes FJ. Increasing insulin resistance is associated with a decrease in Leydig cell testosterone secretion in men. Journal of Clinical Endocrinology and Metabolism 200590 2636-2641. (doi:10.1210/jc.2004-2190)

40 Isidori AM, Caprio M, Strollo F, Moretti C, Frajese G, Isidori A \& Fabbri A. Leptin and androgens in male obesity: evidence for leptin contribution to reduced androgen levels. Journal of Clinical Endocrinology and Metabolism 199984 3673-3680.

41 Houtkooper LB, Lohman TG, Going SB \& Howell WH. Why bioelectrical impedance analysis should be used for estimating adiposity. American Journal of Clinical Nutrition $1996 \mathbf{6 4}$ 436S-448S

42 Horlick M, Arpadi SM, Bethel J, Wang J, Moye J Jr, Cuff P, Pierson RN Jr $\&$ Kotler D. Bioelectrical impedance analysis models for prediction of total body water and fat-free mass in healthy and HIV-infected children and adolescents. American Journal of Clinical Nutrition 2002 76 991-999.

Received 2 March 2015

Revised version received 5 May 2015

Accepted 14 May 2015 